\title{
Large data Age and Academic Innovation of Traditional Chinese Medicine
}

\author{
Zheng Wei ${ }^{1, a}$, Yafeng $\mathrm{Li}^{1, \mathrm{~b}}$, Aihua Zhang ${ }^{1,2, \mathrm{c} \text {, and Junping Zhang }}{ }^{3, \mathrm{~d}^{*}}$ \\ ${ }^{1}$ Henan Academy institute of Traditional Chinese Medicine, Zhengzhou, Henan Province 450000, \\ china \\ a Email:questwz@126.com ${ }^{\mathrm{b}}$ liyafenghntcm@163.com, \\ czhangaihuahntcm@163.com , dzhangjunpinghntcm@163.com \\ * please mark the corresponding author with an asterisk
}

\section{Keywords: Big data age; Chinese medicine; Academic innovation; Feature; Function}

\begin{abstract}
With the modernization and internationalization of Chinese medicine, the modern scientific interpretation of traditional Chinese medicine theory is very urgent, but by the small data and reduction analysis method, to achieve the development and innovation of Chinese medicine academic theory is still struggling. With the development of large data research concept, deep into the field of large-scale research of Chinese medicine to compare the large data and the main features of traditional Chinese medicine, to explore the background of large data in the context of the possibility of innovation in Chinese medicine, trying to change the thinking of Chinese medicine research, To clarify the source and way of large data of traditional Chinese medicine, to realize the integration and application of large data of traditional Chinese medicine, and to find the practical problems in the construction of large data of traditional Chinese medicine, so as to promote the innovation and development of traditional Chinese medicine thinking, TCM basic theory and diagnosis and treatment theory.
\end{abstract}

\section{Introduction}

The era of large data greatly enhances our ability to collect massive amounts of data, gaining a lot of information, predicting the inherent laws of things and phenomena, and forcing the various sectors of modern society to reflect on how to adapt to the trend of large data age to promote self- development. ${ }^{[1]}$ Looking back on the formation of the theoretical system of Chinese medicine, the future trend of the development of Chinese medicine academic development, how to maintain the vitality of Chinese medicine, is the scientific problems facing Chinese medicine. It is difficult to make substantial progress in the theoretical development and academic innovation of traditional Chinese medicine by restricting the research methods of modern scientific reduction. With the help of large data research ideas and methods, this paper analyzes the characteristics and requirements of large data research, combined with the academic characteristics of traditional Chinese medicine, clarifies the sources, construction and analysis methods of traditional Chinese medicine, and explains the practical problems in the study of large data of Chinese medicine. The academic innovation and development of medicine is of great strategic significance. ${ }^{[2]}$

\section{The Enlightenment of the Innovation and Development of Traditional Chinese Medicine in the Era of Big Data}

British data scientist Victor Mary - Schoenberg describes the large data age to deal with the data has three concepts: is the whole data, not random sampling; is a hybrid, not accuracy; is a relationship, not a causal relationship, Reveals the basic characteristics of large data research and requirements ${ }^{[3]}$. In fact, the study of the whole object or the integration of a number of similar independent research data to verify the practicality of the findings in clinical studies have shown, such as evidence-based medicine system evaluation Meta analysis, through a comprehensive collection and integration of a single, similar research literature to obtain higher level of research evidence. The real world research concept emphasizes the safety, efficacy and economic evaluation of drugs in the case of clinical actual 
medication, that is, through the "real world sample" to reflect the real world. Comparative benefit research concept focus on comparing the benefits and risks of different clinical treatment programs, so as to obtain a more informed clinical decision etc., in the research concept has been in line with large data research trend. Relatively speaking, the era of large data research perspective is broader, more open thinking, the impact is more far-reaching. From the logical relationship between research thinking, idea, content and method, the research idea is influenced by the way of thinking, and the research content and method is the external manifestation of the idea ${ }^{[4]}$. Therefore, the biggest enlightenment of the great data age to bring Chinese medicine is to re-examine and change the research and thinking of Chinese medicine that we have and have been cured, to see the academic characteristics of Chinese medicine and to predict the future development trend of Chinese medicine academic.

\section{The Basic Characteristics of the Big Data Age and the Main Features of Traditional Chinese Medicine}

Full Data Model and Overall View. Large data age emphasizes the full data model, that is, the analysis of all the data, rather than random sampling of some of the data to partially reflect the overall characteristics of the data acquisition technology and conditions under the constraints of the move. Therefore, the small data to pursue the accuracy of research in order to improve the credibility of the results of the study, and large data generation and access to the situation has undergone a fundamental change to all the data reflect the overall characteristics is the best way to study, so large data research allows mixed Exist, more concerned about the practicality of the results. The whole view permeates all aspects of Chinese medicine theory and clinical field, emphasizing the unity and integrity of structure and function, that is, the research object as an organic whole, against isolated, one-sided, local interpretation of things and phenomena of the inherent laws and characteristics. So in the research object, research perspective and level of understanding, Chinese medicine and large data research are based in the whole, are to reveal the whole law of things ${ }^{[5]}$.

Related Relations and Functions. The core of the correlation is to quantify the mathematical relationship between the two data values. The correlation between the objects can be expressed by strong correlation and weak correlation ${ }^{[6,7]}$. Usually we are more accustomed to reveal the strong correlation between the two data, Concerned about weak correlation. In contrast to the causes of the correlation, we are more likely to get the results of the correlation, because it is clear that the relationship between things is enough to make us more clearly understand things, more quickly to take interventions. The formation and development of Chinese medicine academic theory does not lack the example of physical structure research, but under the influence of traditional way of thinking, Chinese medicine is difficult to use empirical research method as the main research means, but to oppose unity, universal contact to explain the concept Structure based on the function to clarify the relationship between the two. The formation of the system of Tibetan language is through the observation of external, awakened, Chi, liquid and other physiological and pathological manifestations, to verify the internal organs and the relationship between the function. Therefore, large data research and traditional Chinese medicine research are focused on revealing the correlation between the subjects.

\section{The Possibility of Realizing the Academic Innovation of Traditional Chinese Medicine in the era of Large Data}

Big data age through large data analysis to find the hidden things behind the correlation between things, or things inside the laws and characteristics. Chinese medicine by absorbing Lao Tzu "Tao" cultural thought and connotation, the natural way than the way of the human body, to promote the formation of their own overall concept and development, to promote human survival should conform to the laws of nature and life and rule, and then clear to reveal The Law of Human Life Phenomenon is the Fundamental Task of Traditional Chinese Medicine. Thus, large data age attempts to predict the law of discovery of things, and traditional Chinese medicine through repeated clinical practice and philosophical speculation to explain the basic laws of human life are in common ${ }^{[8]}$. With great data to 
study thinking and methods to promote the academic development and innovation of Chinese medicine, has obvious advantages in the concept. Because Chinese medicine is based on the concept of "taking things as" and "figurative" method, the main body is the product of image thinking. Therefore, although the formation and construction of TCM theory system But the main use of "far from the objects", "close to take the body," the perception and understanding of the way, through the natural society and the body's own physiological and pathological phenomena of long-term observation, the accumulation of clinical practice experience And sublimation, after verification and amendment gradually reached a consensus. Based on individual clinical practice based on the academic theory of Chinese medicine, after thousands of years of long and ups and downs of the formation and development process, although some academic theoretical innovation, but its formation has a certain degree of passive, contingency and uncertainty. In the era of large data of information explosion, the speed of medical information and the number of unprecedented rapid and huge, especially in China's hospital information technology to strengthen, such as the universal application of electronic medical record system, so that access to clinical diagnosis and treatment of large data possible Large medical data sharing, It is possible to improve the efficiency of traditional Chinese medicine research and find new rules and new features of modern Chinese medicine. Finally, it will realize the basic theory of traditional Chinese medicine and medicine, the innovation of clinical diagnosis and treatment, the innovation of clinical evaluation methods of traditional Chinese medicine and the process of promoting the creation of new Chinese medicine.

\section{Large Sources of Data for TCM Innovation}

The large data of Chinese medicine academic innovation mainly comes from several aspects. Clinical medical data: Clinical medical data is the main body of academic innovation of Chinese medicine, mainly from the hospital information system (HIS), such as image archiving and communication systems (PACS), clinical information system (Clinical information system, CIS), laboratory information system (LIS), etc., in which the clinical information system electronic medical records (electronic medical record, EMR) data is particularly important. As the electronic medical records of Chinese medicine hospital contains four medical information and laboratory test data, and the lack of traditional Chinese medicine tongue medical information, the need to integrate the two electronic medical records system data to achieve complementary advantages. (2) Chinese medicine literature data: Including periodicals, conference literature, doctoral thesis, master's thesis, newspapers, patents, standards, books and literature (ancient Chinese medicine, modern medical) and so on. Retrieved CNKI medical and health field literature results show: Chinese medicine, Chinese medicine, traditional Chinese and Western medicine in the field of journal literature up to millions of articles, domestic / international conference documents up to 100,000, and with the development of Chinese medicine, Chinese medicine Deepening the level of scientific research, the amount of Chinese medicine literature will be further rapid growth. Clinical data: mainly including I - IV clinical trial data, spontaneous reporting and active monitoring of safety data, clinical efficacy after the listing of traditional Chinese medicine, safety, economic Sexual research data and so on. (4) Experimental data: Mainly including animal experiments, pre-clinical pharmacology, toxicology experiments and other data. (5) Other data: Such as network information data, drugs, medical equipment sales information data, help to analyze sub-health groups and non-hospital outpatient medical patients with the characteristics of the law.

\section{Analysis on the Key Link of Constructing Large Data of Traditional Chinese Medicine}

reform of traditional Chinese medicine innovation research thinking. In the context of large data, the innovation of Chinese medicine in the background is not the first to collect large data itself. Large data is not a technical approach, but to achieve the premise of large applications, Chinese medicine research thinking change is the key to Chinese medicine academic innovation. It is generally believed that medical experiment detection is a necessary means to determine the occurrence of disease, but based on massive network search information data to build mathematical model, can accurately predict 
the influenza A H1N1 influenza outbreak of the epidemic time and region, Therefore, with the help of large data network data can predict the disease. Therefore, the study of traditional Chinese medicine innovation should not only focus on animal experiments and clinical trials, to demanding the level of research, level and research technology of advanced nature, should also focus on the formation of Chinese medicine academic theory roots, the use of clinical medical data innovation Chinese medicine academic theory, and Cultivate the ability to discover the academic laws of Chinese medicine from other modern information data.

Chinese medicine large data construction and access. Large data of Chinese medicine can be derived from network information, electronic information and other non-Chinese medicine data, but more importantly, through the large Chinese medicine to sum up the theory of traditional Chinese medicine theory and evolution of the law. At present, China's large data of Chinese medicine in addition to the journal data, the lack of large-scale, standardized database, although the actual production of Chinese medicine diagnosis and treatment data is very complex, but through a wide range of multi-level information database construction, to be able to save And applied to large data analysis. According to 2007, the clinical information system of 3765 hospitals in China showed that the application rate of LIS system was $26.43 \%$ and the application rate of PACS system was only $9.00 \%$, which indicated that the clinical medical information Construction and application are still in the initial stage of development. In addition, the acquisition of large Chinese medicine data is not easy, individual behavior is difficult to obtain enough large data and get a big application, timely and orderly information and open policy guidance from top to bottom is extremely necessary. In the large data acquisition there are many details of the problem, such as the standardization of Chinese medicine terminology applications, patients with medical information confidentiality and ethical principles, which is an important factor affecting the construction of large data.

Large Selection of Chinese Medicine Data. Single TCM data is difficult to meet the needs of large data research on the amount of data and data, but still can not be considered the greater the amount of data the better, too large data on behalf of too much processing time and workload, and the accuracy of the results and Not necessarily with the infinite expansion of the amount of data has been significantly improved, so the choice of data is large data acquisition and integration must be taken seriously when the important part

Integration and Application of Large Data of Traditional Chinese Medicine. Traditional Chinese medicine data system mostly text, images and other unstructured data, the real world of the various information generated by non-structured forms exist, but also for different heterogeneous data integration, for large data construction and analysis of the band To a huge challenge, only multi-disciplinary technology is a combination of integration and analysis of large data effective means. Such as data visualization technology can visually display and understand the relationship between large data research and the law; improved data mining and machine learning technology Data processing technology includes data cleansing, data integration and online analysis processing. The online analytical processing (OLAP) has the ability to aggregate, merge and aggregate functions, and to view information from different perspectives. [6]. Due to the large data research in the ascendant, especially in the field of Chinese medicine research, the lack of corresponding research base and examples, based on massive metadata, the use of data warehousing, data mining technology to solve the complex integration between heterogeneous databases, Is conducive to large data research carried out, and large data cross, integration and analysis methods still need to practice exploration and verification.

\section{Summary}

As people deal with the transformation of data thinking patterns, the ability to collect, store, transmit and analyze data, in the face of large and complex information, we can stand at unprecedented levels of research, dig and predict with a broad perspective of overall analysis Things and the phenomenon of the overall law, and effectively improve the work and life efficiency. For the academic innovation of traditional Chinese medicine, the analysis of large data can meet the needs of the sustainable development of Chinese medicine, but the scope and extent of the construction of large data 
construction of Chinese medicine is very different. The process of obtaining, processing and analyzing Chinese medicine large data still exists. Kind of concept and technical deficiencies, in order to truly realize the large application of large Chinese medicine data, still need the theory and practice of continuous attempt and discussion.

\section{References}

[1] Vicini P, Fields O, Lai E, et al. Precision medicine in the age of big data: The present and future role of large-scale unbiased sequencing in drug discovery and development. Clin Pharmacol Ther. 2016. 99(2): 198-207.

[2] Lee CS, Sengupta D, Bhargavan-Chatfield M, Sickles EA, Burnside ES, Zuley ML. Association of Patient Age With Outcomes of Current-Era, Large-Scale Screening Mammography: Analysis of Data From the National Mammography Database. JAMA Oncol. 2017. 3(8): 1134-1136.

[3] Åkerstedt T, Hallvig D, Kecklund G. Normative data on the diurnal pattern of the Karolinska Sleepiness Scale ratings and its relation to age, sex, work, stress, sleep quality and sickness absence/illness in a large sample of daytime workers. J Sleep Res. 2017 .

[4] Smith RL, Haslemo T, Refsum H, Molden E. Impact of age, gender and CYP2C9/2C19 genotypes on dose-adjusted steady-state serum concentrations of valproic acid-a large-scale study based on naturalistic therapeutic drug monitoring data. Eur J Clin Pharmacol. 2016. 72(9): 1099-104.

[5] Roux PP, Thibault P. The coming of age of phosphoproteomics--from large data sets to inference of protein functions. Mol Cell Proteomics. 2013. 12(12): 3453-64.

[6] Shen X, Liu H, Hu Z, Hu H, Shi P. The relationship between cerebral glucose metabolism and age: report of a large brain PET data set. PLoS One. 2012. 7(12): e51517.

[7] Van der Elst W, Van Boxtel MP, Van Breukelen GJ, Jolles J. The Stroop color-word test: influence of age, sex, and education; and normative data for a large sample across the adult age range. Assessment. 2006. 13(1): 62-79.

[8] Kroke A, Schulz M, Hoffmann K, Bergmann MM, Boeing H. Assignment to menopausal status and estimation of age at menopause for women with missing or invalid data--a probabilistic approach with weighting factors in a large-scale epidemiological study. Maturitas. 2001. 40(1): 39-46. 